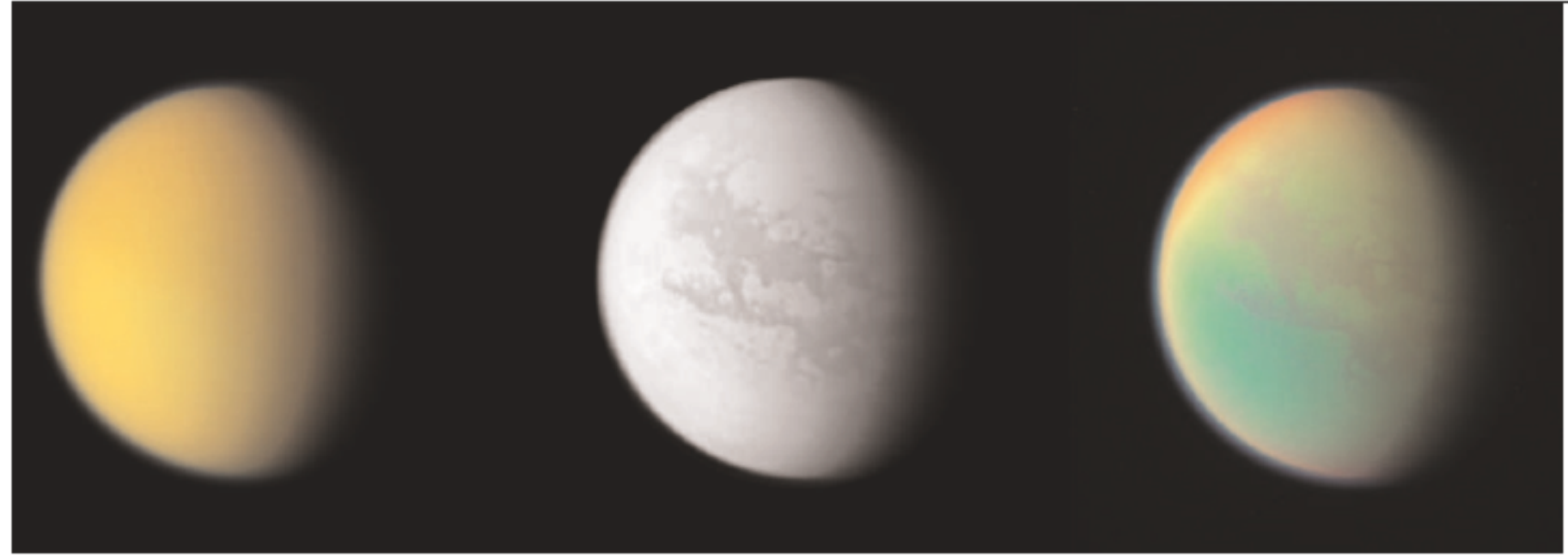

\title{
Titant tapping the flood of data
}

\section{The first analyses of the Huygens mission to Titan are published this week. Mark Peplow charts the satellite's transition from fogbound moon to familiar landscape, and finds out why scientists long to return.}

It is easy to forget that just over a year ago, Titan was one of the most mysterious objects in our Solar System. Larger than the planet Mercury, Saturn's largest satellite is the only moon known to have a dense atmosphere. And during years of telescopic observations, Titan has remained hidden beneath its thick hydrocarbon smog, a well-wrapped gift held tantalizingly out of reach.

But since the Cassini-Huygens mission arrived at the ringed planet last year, scientists have been clearing up Titan's mysteries at a tremendous pace. "Before Cassini-Huygens, Titan was the largest unexplored surface in the Solar System," says Ralph Lorenz, a planetary scientist from the University of Arizona, Tucson. "Now scientists are just trying to stand up against the fire hose of information."

Cassini has made nine close fly-bys of the moon, peering through the murk with its radar and infrared sensors, but some of the most exciting details have come from the Huygens lander. This probe hitched a ride halfway across the Solar System with Cassini before descending to Titan's surface on 14 January, and its first results are published online by Nature this week (T. Owen Nature doi:10.1038/ 438756a; 2005).

Titan attracts scientists because its methane and nitrogen atmosphere is reminiscent of a primordial, prebiotic Earth. Some hope that understanding Titan's chemistry might reveal how the first molecules oflife were formed on our own planet.

The methane was first spotted in 1944 when astronomer Gerard Kuiper studied the spectrum of Titan's atmosphere. But fleeting visits by the Voyager probes in 1980 and 1981 revealed that the atmosphere was actually mostly nitrogen, squeezed to 1.6 times the Earth's atmospheric pressure at the surface.

Along with a chilly surface temperature of $-180^{\circ} \mathrm{C}$, this fuelled speculation that liquid hydrocarbon oceans might cover the moon. "If you read the papers beforehand you saw predictions for almost everything imaginable," says John Zarnecki from the Open University, Milton Keynes, UK, who heads Huygens' surface-science team. One of the strongest possibilities was that Titan was the only place in the Solar System besides Earth where rain fell on a solid surface.

\section{Reach for the moon}

The only way to find out was to pay a visit. The Cassini mother ship and Huygens probe were built in an unprecedented collaboration between NASA, the European Space Agency and the Italian Space Agency. After a sevenyear trek to Saturn, Cassini made its first close pass over Titan on 26 October 2004, and saw fuzzy patterns on the surface that seemed to bear the marks of flowing material.

When Huygens dived through the atmosphere three months later, the pictures became crystal clear, revealing networks of deep channels carved into the landscape. ${ }^{\alpha}$ The striking thing is how much they look like fluvial channels on Earth," says Larry Soderblom of the US Geological Survey in Flagstaff, Arizona. Some channels are 50-100 metres deep, and provide compelling evidence that rain has fallen on the planet. Other gorges and canyons suggest that lava-like flows and springs have belched liquid from Titan's interior.

This is hard evidence that Titan is geologically active, and has the methane equivalent of a hydrological cycle, with material travelling from surface to skies and back again. "I call it the methalogical cycle," says Sushil Atreya, an expert in planetary chemistry at the University of Michigan in Ann Arbor. "Methane really controls the whole atmosphere of Titan."

The probe confirmed that ultraviolet light from the Sun breaks upper-atmosphere methane into fragments, which then combine to form larger organic molecules, such as polyaromatic hydrocarbons. These condense into Titan's characteristic haze of aerosol particles.

Gradually, the aerosols fall from the atmosphere. "Effectively it's grit, snowing down on to the surface," says Soderblom, who is one of the mission's interdisciplinary scientists. According to pictures taken during Huygens' descent, this dark snow collects in river basins and lakes, as though it has been washed off the brighter hills.

Scientists are now certain that the atmosphere is $5 \%$ methane: this is not quite enough to produce frequent showers or sustain large lakes, but raises the possibility of occasional monsoons. There is no evidence of oceans, which were thought to be a likely source for the atmosphere's methane - the compound only survives for 10 million to 100 million years in the atmosphere before being broken down.

Carbon isotope measurements also rule out a biological source for the methane - at least 
one that is anything like life on Earth. Instead, Atreya suspects that the methane may be generated deep beneath the surface by geological processes that digest carbon dioxide and other organic compounds. A 30-kilometre-wide dome on the surface could be one gate to this underworld - it is thought to be a 'cryovolcano', periodically oozing a chilly mixture of water, ammonia and gases from the moon's innards. And this cold lava could bring up methane from below the surface.

Although the data are impressive, for Zarnecki the highlight of Huygens' trip is the fact that the probe arrived successfully - it was the most distant controlled landing ever made. "Even though I was part of the team that designed Huygens to be as robust as possible, I was amazed.

The probe touched down safely on a surface with the consistency of wet sand, its heat throwing up a gentle plume of methane and more complex organic molecules. Images showed Huygens surrounded by smooth pebbles sitting in gullies, apparently hollowed out by flowing liquid. "The surface was a big surprise, it was totally new information," says Hasso Niemann of NASA's Goddard Space Flight Center in Greenbelt, Maryland. Niemann, who ran Huygens' mass spectrometer,

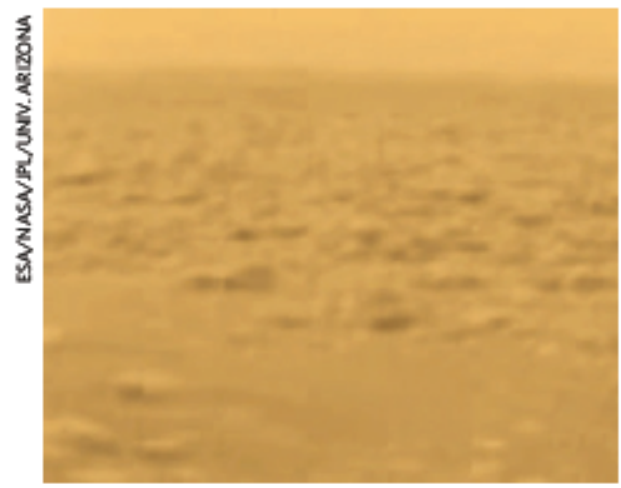

Cassini snapped multiple views of Titan (top, left), but these crystal clear images of the surface were Huygens' reward for plunging below the fog.

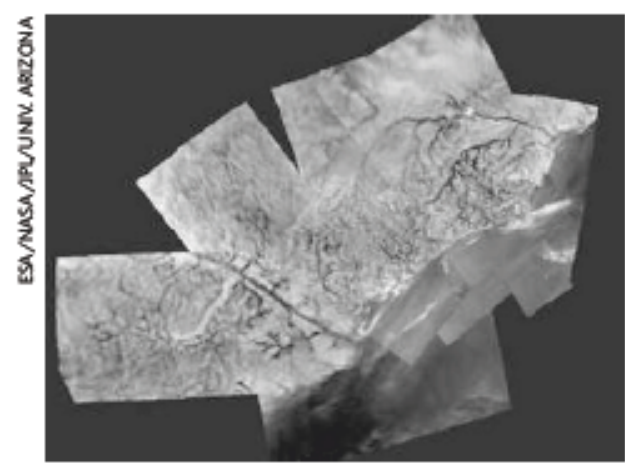

adds that the team still doesn't know for sure what the pebbles are made of.

Another question unlikely to be solved soon is whether Titan's chemical soup can make molecules essential for life, such as amino acids; Huygens was not set up to identify chemicals that accurately. The moon's surface is one of the most promising places in the Solar System for finding these molecules, because it hosts the products of chemistry, which have drifted down from the haze above. Add liquid water and ammonia from cryovolcanoes, and you have an ideal lab for studying how the molecules of life might form over thousands of years. "Nobody can do that experiment on Earth," says Lorenz. That makes a return visit extremely attractive.

\section{Future forays}

As well as being a member of the surfacescience team, Lorenz chairs NASA's Outer Planets Assessment Group, which prioritizes science goals for missions to the outskirts of the Solar System. The group ranks a return to Titan as the second most important mission to work on, after a trip to Jupiter's ice-covered moon Europa. Titan's low gravity and thick atmosphere make a robotic airship an attractive prospect, adds Zarnecki, because it could hop around many different sites easily.

Such a mission may take ten years of planning, followed by the seven-year journey to Saturn. But in the meantime Cassini still has at least 36 fly-bys of Titan to complete. Its radar has only mapped a small percentage of the surface, but Soderblom thinks it may be able to cover up to a quarter of the alien landscape. More detail should also come out when Huygens' and Cassini's data are compared more closely, and pictures from the descent are tied to radar images from orbit.

“The biggest question is what energy source drives Titan's surface geology and weather, says Soderblom. The Sun provides very little light, and radioactive decay and internal movement caused by Titan's orbit around Saturn don't seem to add much power.

Soderblom suggests that although Titan's sparsely cratered surface looks young, it could be hundreds of millions of years old. The atmosphere is thick enough to burn up most meteorites before they reach the surface, and drifting dunes of hydrocarbon dust may obscure craters. If it is older than it looks, weak sunlight may be just enough to turn Titan's methalogical cycle in slow motion. There could still be powerful monsoons once every few centuries, points out Lorenz. Any future probes may be well advised to pack an umbrella. 